\title{
Mechanism of the Oxidation of Heptafulvenes to Tropones Studied by Online Mass Spectrometry and Density Functional Theory Calculations
}

\author{
Sofia M. E. Nilsson, ${ }^{\dagger}$ Henning Henschel, ${ }^{\ddagger}, \S \odot$ Gianmario Scotti, ${ }^{\dagger}$ Markus Haapala, ${ }^{\dagger}$
} Alexandros Kiriazis, ${ }^{\dagger}$ Gustav Boije af Gennäs, ${ }^{\dagger}$ Tapio Kotiaho, ${ }^{\dagger}, l_{\odot}$ and Jari Yli-Kauhaluoma*, ${ }^{\dagger}$

${ }^{\dagger}$ Drug Research Program, Division of Pharmaceutical Chemistry and Technology, Faculty of Pharmacy, P.O. Box 56 (Viikinkaari 5 E), FI-00014 University of Helsinki, Helsinki, Finland

${ }^{\ddagger}$ Research Unit of Medical Imaging, Physics and Technology, University of Oulu, P.O. Box 5000 (Aapistie 5 A), FI-90220 Oulu, Finland

${ }^{\S}$ Medical Research Center, University of Oulu and Oulu University Hospital, P.O. Box 5000 (Aapistie 5 A), FI-90220 Oulu, Finland

"Department of Chemistry, Faculty of Science, P.O. Box 55 (A.I. Virtasen Aukio 1), FI-00014 University of Helsinki, Helsinki, Finland

Supporting Information

ABSTRACT: We have identified the most likely reaction mechanism for oxidizing heptafulvenes to the corresponding tropones by experimental and theoretical investigations. The experimental studies were done by coupling a three-dimensional printed miniaturized reactor with an integrated electrospray ionization needle to a mass spectrometer. Using the experimentally observed ions as a basis, nine alternative reaction pathways were investigated with density functional theory calculations. The lowest energy reaction pathway starts with the formation of an epoxide that is opened upon the addition of a second equivalent of the oxidizing species meta-chloroperoxybenzoic acid. The adduct formed then undergoes a Criegee-like rearrangement to yield a positively charged hemiketal, which on deprotonation dissociates into acetone and tropone. Overall, the reaction mechanism resembles a Hock-like rearrangement.

\section{INTRODUCTION}

Tropones and troponoids are interesting compounds due to their anticancer properties, ${ }^{1,2}$ antimicrobial activity, ${ }^{3,4}$ and antifungal effects. ${ }^{5}$ The oxidation of the exocyclic carboncarbon double bond of a heptafulvene by meta-chloroperoxybenzoic acid ( $m$ CPBA) provides an unusually mild method for obtaining tropones. ${ }^{6,7}$ Understanding the mechanism of this reaction would allow for optimizing the reaction conditions to obtain the maximal yield of the desired product and minimal formation of byproducts. A strong tool for experimentally studying the reaction mechanisms is a microreactor coupled online with mass spectrometric (MS) detection. ${ }^{8}$ In comparison to related macroscopic systems, microreactors usually require smaller amounts of reactants and improve heat and mass transfer, the latter resulting in shorter reaction times. ${ }^{9}$ These characteristics, together with the possibility of automated sampling offered by (miniaturized) systems coupled online with a detector, can be exploited for reaction mechanism studies. The high sensitivity of MS detection and its mass-to-charge $(\mathrm{m} / z)$ ratio-based identification of reaction intermediates and products, which is further supported by multiple stage $\mathrm{MS}\left(\mathrm{MS}^{\mathrm{n}}\right)$ data, facilitate the identification of various species, ${ }^{10}$ thus making MS an excellent choice for reaction mechanism studies. Additionally, since MS detection enables very rapid response times, ${ }^{11}$ it is good for detecting intermediates of interest. Examples highlighting the capability of online MS to detect short-lived reaction intermediates include the Negishi reaction, ${ }^{12}$ microdroplet studies of the cycloaddition between diethyl azodicarboxylate and quadricyclane, ${ }^{13}$ as well as of hydrazone formation, ${ }^{14}$ and monitoring of visible-light-mediated $[3+2]$ annulation. ${ }^{15}$ Microreactors and flow chemistry systems combined with MS have been used for probing reaction mechanisms. ${ }^{16}$

MS provides only limited structural information, and the species observed may not be unambiguously identifiable by MS. In these scenarios, computational chemistry can be used in synergy with the experimental results. Using computational chemistry to investigate the potential energy profiles of potential reaction pathways, the alternative mechanism with the lowest energy barrier can be identified. The combination of

Received: August 5, 2019

Published: September 27, 2019 
MS and computational chemistry for resolving mechanisms has been applied for a number of organic ${ }^{17-19}$ and organometallic $^{20-23}$ reactions. Nevertheless, the combination of computational chemistry and microreactors coupled online with MS detection for probing reaction mechanisms seems to be a concept hitherto unexplored.

Here, a three-dimensional (3D) printed miniaturized reactor fabricated of the chemical-resistant polymer polypropylene ${ }^{24}$ was combined with an ion trap MS operated in the positive electrospray ionization (ESI) mode to study the oxidation of heptafulvene $\mathbf{1}$ to tropone $\mathbf{5}$ (Scheme 1) online. In addition,

Scheme 1. Meta-Chloroperoxybenzoic Acid (mCPBA)Mediated Oxidation of Heptafulvene 1 into Tropone 5

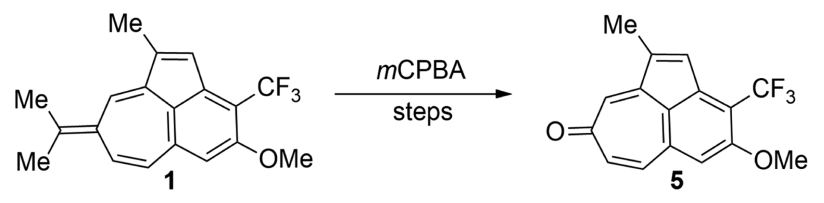

density functional theory (DFT) calculations were performed (M06-2X/6-311++G(d,p)) to assess the various mechanisms that would explain the appearance of the species observed. The energies obtained from these calculations are given as Gibbs free energies, unless explicitly noted otherwise.

\section{RESULTS AND DISCUSSION}

Figure 1 shows typical experimental results in terms of extracted ion profiles (EIPs) of the ions of interest and an averaged mass spectrum at the end of the experiment. The experiment was performed as follows: at $t=0$, acquisition of mass spectra was started (and continued during the whole experiment); immediately after this, compound $\mathbf{1}$ from one syringe and $m \mathrm{CPBA}$ (both dissolved in acetonitrile containing 0.1 vol \% formic acid) from another syringe were infused into the miniaturized reactor at flow rates of $125 \mu \mathrm{L} / \mathrm{min}$ each. At 1 min, the miniaturized reactor, which has a volume of approximately $250 \mu \mathrm{L},{ }^{24}$ is filled and the infusion of reactants is stopped. At this time point, the integrated stir bar of the miniaturized reactor is activated, and optimization of the position of the reactor in front of the capillary extension of the MS is started until a stable electrospray is obtained at approximately $2.5 \mathrm{~min}$, as shown in Figure 1a. This is observed as an increase in the EIP intensities. Additional mass spectra for the experiment shown here, as well as the averaged mass spectra and EIPs from other experiments, are presented in the Supporting Information, S2.1-2.2. The results of these experiments are in accordance with those presented in Figure 1.

The most interesting ions observed in the mass spectrum (Figure $1 \mathrm{~b}$ ) are $m / z 293,319,335$, and 351 (the compound and ion structures are given in Figure 1a and Scheme S5). These species were initially identified by the analysis of the pure starting material (1) and the tropone reaction product (5, synthesized and purified offline) using ion trap MS and liquid chromatography-mass spectrometry (LC-MS). In addition, the change in EIP intensities of the reaction species as a function of time and $\mathrm{MS}^{\mathrm{n}}$ studies (Supporting Information, S2.3) aided the identification of these species. The ion at $\mathrm{m} / z$ 293 was assigned as the protonated tropone reaction product $[\mathbf{5}+\mathrm{H}]^{+}, m / z 319$ the protonated heptafulvene $[1+\mathrm{H}]^{+}$, and $\mathrm{m} / \mathrm{z} 335$ the protonated first intermediate (epoxide) of the reaction $[2+\mathrm{H}]^{+} /[9]^{+}$. For the ion at $m / z 351$, structures of $[\mathbf{4}]^{+},[\mathbf{8}+\mathrm{H}]^{+}$, and $[\mathbf{S 1}]^{+}$(Scheme 2) were investigated computationally. As can be seen from the EIPs (Figure 1a), already at the start of the experiment, some ions were formed via oxidation of heptafulvene $1(\mathrm{~m} / \mathrm{z} 319)$, particularly $\mathrm{m} / \mathrm{z}$ 335 , but $m / z 351$ and $m / z 293$ were also present. These oxidized species of $\mathbf{1}$ were also observed when analyzing pure $\mathbf{1}$ with liquid chromatography (LC)-ESI-MS (further details in the Experimental Section and S1.1.2). Since only one chromatographic peak for compound 1 was seen in the LCMS chromatogram, and nuclear magnetic resonance (NMR) analysis of $\mathbf{1}$ indicated that it was pure (S1.1.1), we attributed the presence of these ions at the start of the experiment to the fact that analytes can be oxidized during ESI. ${ }^{25}$ Formation of the reaction products and intermediates and their structural assignments are discussed in further detail together with the DFT calculation results. The mass spectrum in Figure $1 \mathrm{~b}$ also shows ions at $m / z 276,363$, and 380. These ions are discussed in the Supporting Information, Section S2.2.

Based on the mass spectrometrically detected ions and their temporal profiles, the reaction pathways for DFT investigation were drafted (main pathways are shown in Scheme 2; additional considered pathways can be found in the Supporting Information, Section 3.1). The color coding of the molecules presented in Scheme 2, as well as of the plots in Figure 2, represents the alternative reaction routes with different intermediates and/or transition states (TSs) and thus energy barriers. The structures depicted in black are part of all of the reaction pathways considered. The reaction pathway with the lowest overall reaction barrier, the blue acid-catalyzed exoxyclic Criegee rearrangement pathway, is discussed first, and subsequently two additional reaction pathways with sequentially higher energy (the turquoise endocyclic Criegee rearrangement pathway and the pink 1,2-dioxetane formation pathway), accounting for the occurrence of the ion at $m / z 351$, are presented below. Additional higher energy barrier reaction pathways are presented in detail in the Supporting Information, Section 3. A summary of the reaction pathway names, the color coding for each reaction pathway, and species of each pathway are given in Table 1.

All pathways (Schemes 2 and S5, Figure 2, and Table 1) start with the mCPBA oxidation of the exocyclic carboncarbon double bond of heptafulvene $\mathbf{1}$ into an epoxide moiety of 2 via transition state TS1 (Figure 3 ). In its protonated form $\left([2+\mathrm{H}]^{+} /[9]^{+}\right)$, this can be observed as an ion with $m / z 335$. The epoxide species 2 can subsequently react with a second equivalent of $m \mathrm{CPBA}$ through a bimolecular nucleophilic substitution $\left(S_{\mathrm{N}} 2\right)$ reaction with concerted proton transfer between the epoxide oxygen of 2 and the peroxycarboxyl moiety of $m$ CPBA (TS2, Figure 3), producing the intermediate 3 .

If intermediate 3 follows the blue reaction pathway and undergoes an acid-catalyzed exocyclic Criegee rearrangement through TS3 (Figure 3) after the addition of a metachlorobenzoic acid ( $m \mathrm{CBA})$ molecule, it results in the formation of $[4 \cdot 2 \mathrm{mCB}]$. Dissociation of this complex yields 4 , with a positive charge delocalized over the ring system. This ion corresponds to $\mathrm{m} / z$ 351. Tandem MS analysis of the $\mathrm{m} / z$ 351 precursor ion yields fragments, which can be explained based on the structure of $[\mathbf{4}]^{+}$(Table S1, Figure S22, and Scheme S3a), suggesting that $\mathrm{m} / z 351$ in the mass spectrum (Figure $1 \mathrm{~b}$ ) has the structure of 4 . Following deprotonation, 4 dissociates barrierless into tropone product $\mathbf{5}$ and acetone. The 

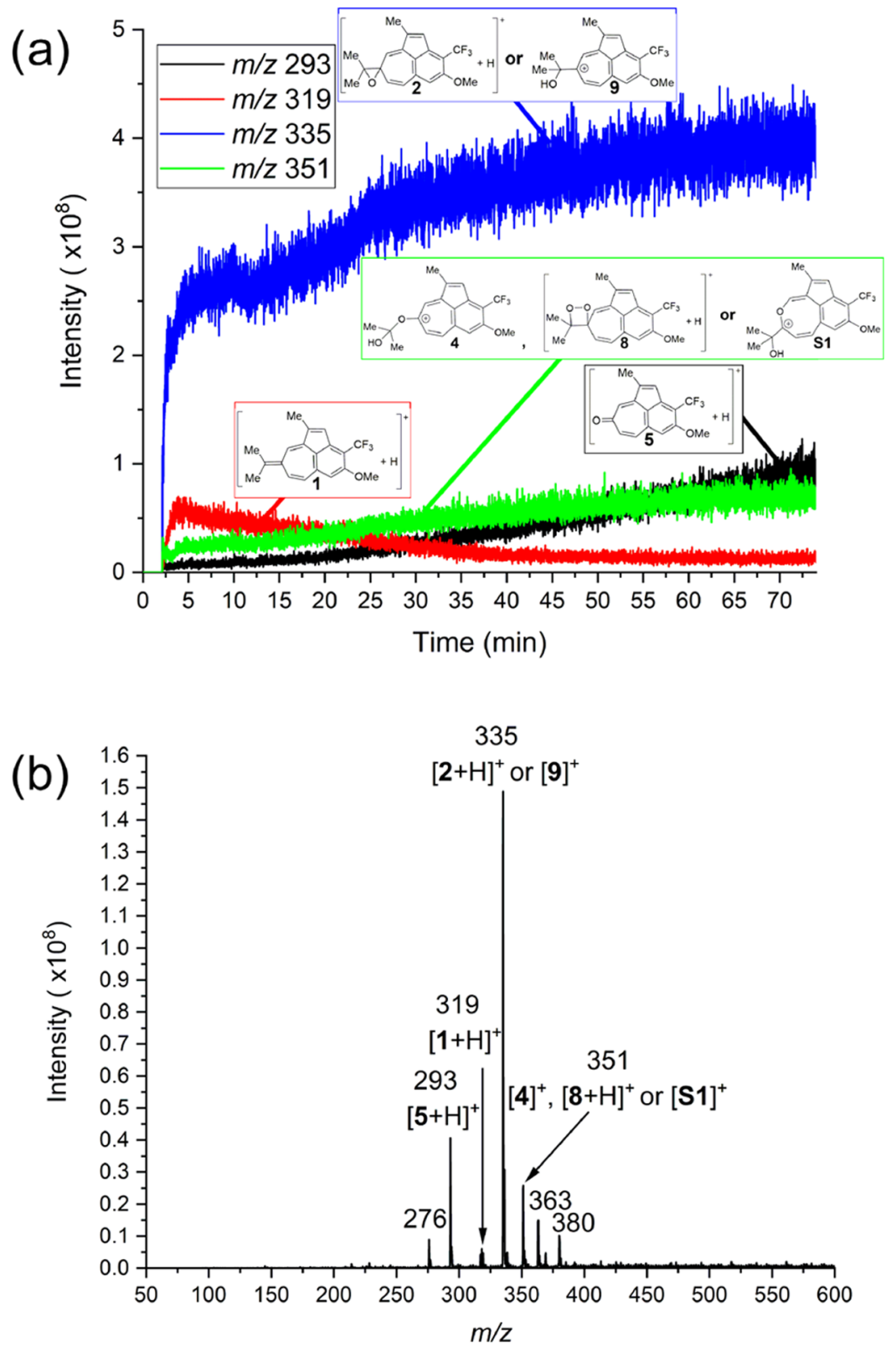

Figure 1. (a) Extracted ion profiles of mass-to-charge ratio $m / z 319\left([\mathbf{1}+\mathrm{H}]^{+}\right.$, red $), m / z 335\left([2+\mathrm{H}]^{+}\right.$or $[9]^{+}$, blue $), m / z 351\left([4]^{+}\right.$, $[8+\mathrm{H}]^{+}$, or $[\mathbf{S 1}]^{+}$, green $)$, and $m / z 293\left([5+\mathrm{H}]^{+}\right.$, black) obtained during the online reaction monitoring. (b) Averaged mass spectrum at $71-72$ min showing the full $\mathrm{m} / z$ range measured. The same spectrum, but with an inset zoomed in at the $\mathrm{m} / z$ range of interest, is shown in Figure S17.

blue acid-catalyzed exocyclic Criegee rearrangement pathway, proceeding through TS3 (Schemes 2 and S6, Figures 2, and S26), has the lowest overall energy barrier, i.e., is the most favored pathway.

Another possible explanation for the detection of $\mathrm{m} / z 351$ arises from the turquoise endocyclic Criegee rearrangement pathway (Schemes 2 and S7 and Figure S27) as characterized by TS4. Following this pathway, $m / z 351$ would instead have the structure of an oxocine ring, which cannot easily react further in any way relevant to this study. This reaction pathway deviates at the aforementioned compound 3. The mechanism of formation of $\mathbf{S 1}$ is essentially the same as that for the formation of 4 , i.e., a Criegee rearrangement. However, while the exocyclic reaction can benefit from catalysis by one additional acid molecule, the geometry of the TS of the endocyclic reaction does not allow for this. The compound formed, S1, is positively charged with an $\mathrm{m} / z$ of 351 . However, investigation of the precursor ion $\mathrm{m} / z 351$ with tandem MS shows a fragment at $\mathrm{m} / z 276$, which is formed by a loss of 75 from $m / z 351$ (Table S1, Figure S22, and Scheme S3). This product ion cannot be as easily explained, based on the precursor ion having the structure of $[\mathbf{S 1}]^{+}$, as is possible from the structure of the precursor ions $[4]^{+}$or $[8+\mathrm{H}]^{+}$. However, it is important to keep in mind that the reaction through the acid-catalyzed exocyclic Criegee rearrangement pathway (via TS3) is dependent on the $m$ CBA concentration because the progress through TS3 requires one additional equivalent of $m \mathrm{CBA}$, while this is not the case for TS4.

In addition to the above described reactions, peroxy ester 3 could alternatively pursue the pink 1,2-dioxetane reaction pathway to yield a species with the $m / z 351$. Here, 3 reacts through TS6 (the dark green 1,2,4-trioxane cycloreversion 
Scheme 2. Three Main Reaction Pathways Evaluated by Density Functional Theory Calculations ${ }^{a}$

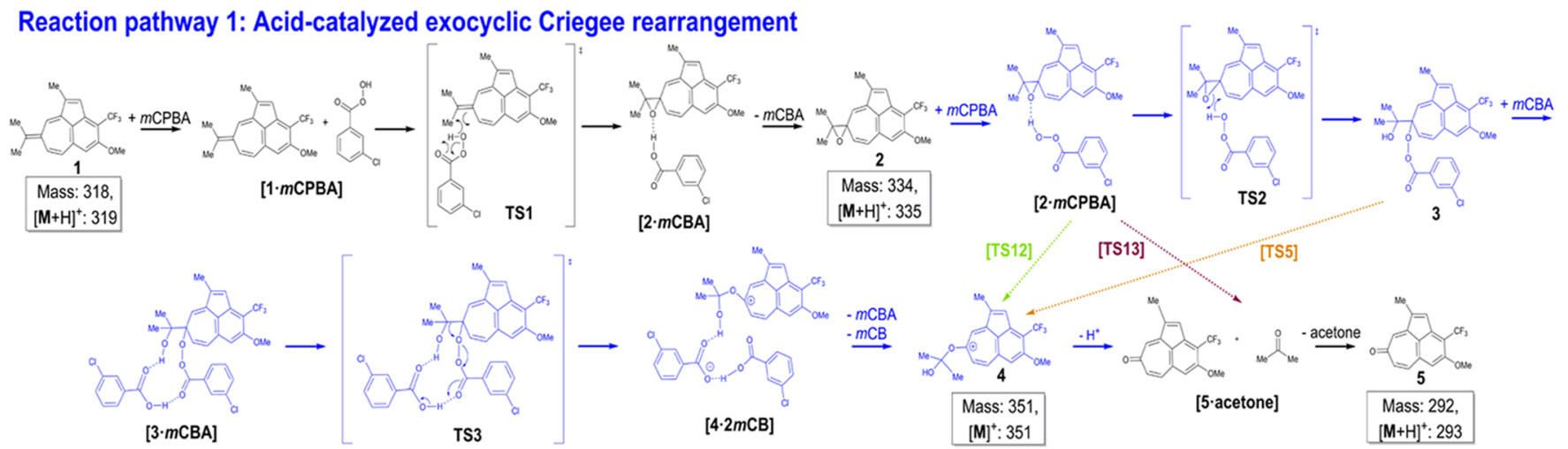

Reaction pathway 2: Endocyclic Criegee rearrangement

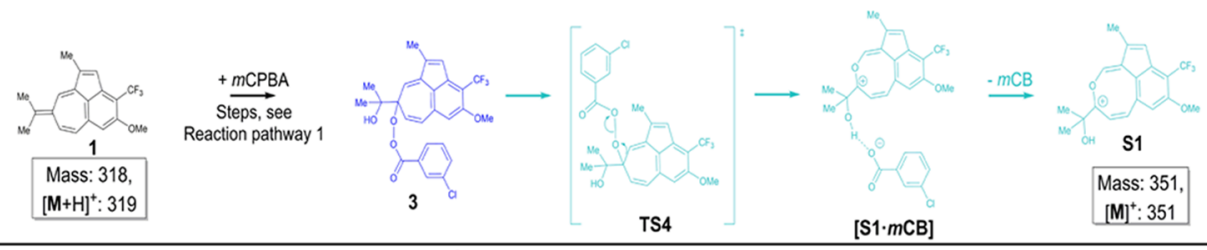

Reaction pathway 3: 1,2-Dioxetane formation

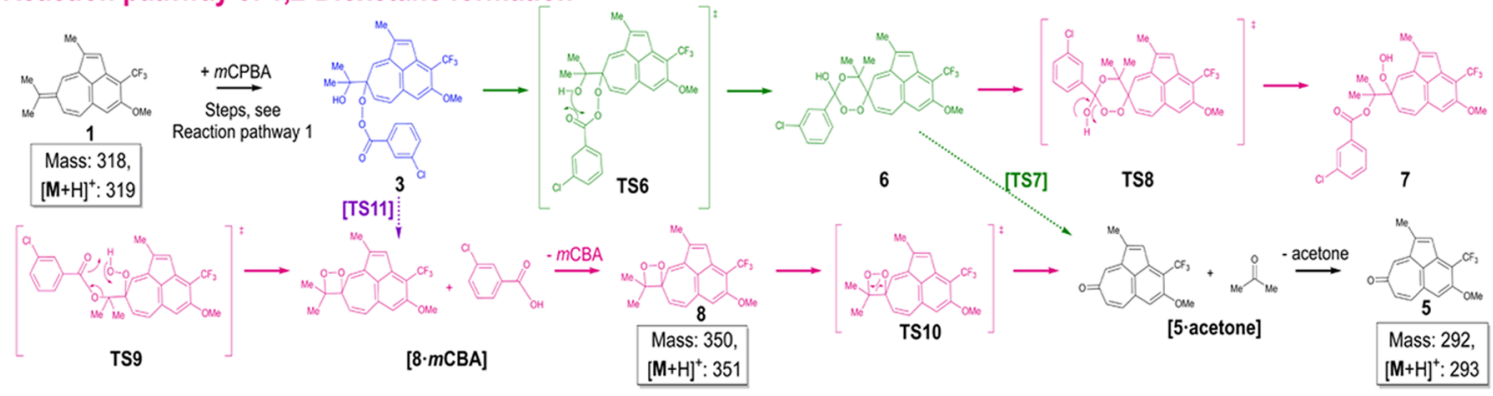

${ }^{a}$ Compound 2 can also, by protonation, be converted directly to 3 via 9 (red tropylium pathway). Additional investigated reaction pathways, presented in further detail in Table 1 and the Supporting Information, are briefly represented with dashed arrows. Mass values of the mass spectrometrically observed species and the corresponding ions with $m / z$ values are presented in the framed boxes. TS1-13=transition states $1-$ $13, m \mathrm{CPBA}=$ meta-chloroperoxybenzoic acid, $m \mathrm{CB}=$ meta-chlorobenzoate, and $m \mathrm{CBA}=$ meta-chlorobenzoic acid.

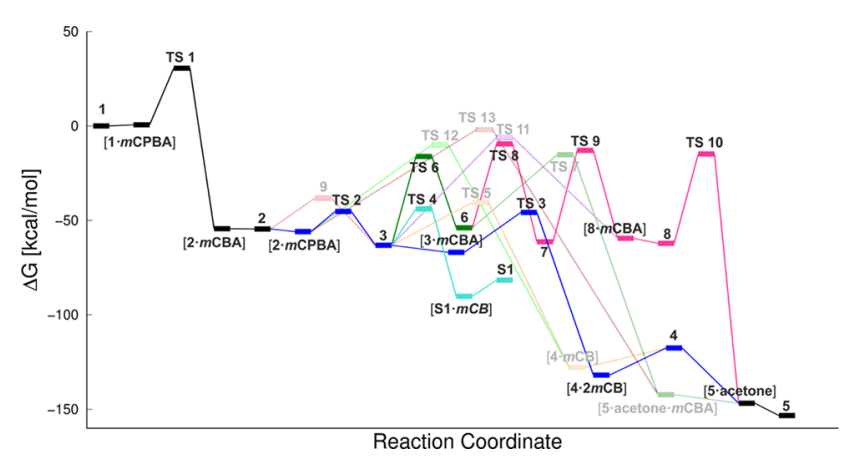

Figure 2. Overview of the Gibbs free energies of each mechanistic step, calculated at the M06-2X/6-311++G(d,p) level. The colors of the plots correspond to the respective colors of the structures proposed in Scheme 2. Reaction pathways not discussed in detail in the main manuscript are shown with faded color. TS $1-13=$ transition states $1-13, m \mathrm{CPBA}=$ meta-chloroperoxybenzoic acid, $m \mathrm{CB}=$ metachlorobenzoate, and $m \mathrm{CBA}=$ meta-chlorobenzoic acid.

route, characterized by TS7, Scheme S9 and Figure S29) by a nucleophilic attack of the hydroxy oxygen atom on the metachloroperoxybenzoate $(m \mathrm{CPB})$ moiety's carboxyl carbon atom. This results in a spiro compound, 6 , which by undergoing a ring opening (TS8), joins the pink 1,2-dioxetane reaction pathway (characterized by $\mathbf{8}$, Scheme S10 and Figure S30), and through TS9, spiro-1,2-dioxetane 8 is formed.

When 8 undergoes $[2+2]$-cycloreversion (TS10), the tropone product $\mathbf{5}$ and acetone are formed simultaneously. It should be noted that we have only studied this typically thermally forbidden reaction step in the singlet ground state. This, however, we found to follow a concerted mechanism, and were not able to locate any low-energy TS corresponding to the formation of a biradical structure, as described by, e.g., Farahani et al. $^{26}$ for 1,2-dioxetane. Therefore, the energy given for this step should be treated as an upper limit rather than a definite value. In any case, this does not affect our conclusions, since TS10 is not the TS highest in energy on this specific reaction pathway. Furthermore, since the energy of this reaction pathway is higher than that of the acid-catalyzed exocyclic Criegee rearrangement pathway, the structure of $\mathrm{m} / z$ 351 does, in spite of its proposed fragmentation pattern being a good match for both structures $[4]^{+}$and $[8+\mathrm{H}]^{+}$(Scheme S3), likely belong to $[4]^{+}$and not to $[8+\mathrm{H}]^{+}$.

A tentative mechanism for the oxidation of heptafulvenes to tropones with $m \mathrm{CPBA}$, involving a direct transition from 3 to 5, without any further intermediates, has been proposed previously. ${ }^{6}$ Moreover, a study of a related reaction, in which the $m$ CPBA oxidation of a 4-hydroxy analogue of heptafulvene 
Table 1. Summary of the Reaction Pathways Investigated by Density Functional Theory (Figure 2 and Supporting Information, Section 3), Ordered Based on Descending Energy Barrier ${ }^{a}$

\begin{tabular}{|c|c|c|}
\hline reaction pathway & $\begin{array}{l}\text { pathway } \\
\text { color }\end{array}$ & $\begin{array}{l}\text { species included in the } \\
\text { pathway, other than the } \\
\text { shared ones }\end{array}$ \\
\hline $\begin{array}{l}\text { acid-catalyzed exocyclic Criegee } \\
\text { rearrangement (Scheme S6 and } \\
\text { Figure S26) }\end{array}$ & blue & $\begin{array}{l}{[2 \cdot m \mathrm{CPBA}], \mathrm{TS} 2,3,[3 \cdot} \\
m \mathrm{CBA}], \mathrm{TS} 3,[4 \cdot 2 \\
m \mathrm{CB}], 4\end{array}$ \\
\hline $\begin{array}{l}\text { endocyclic Criegee rearrangement } \\
(\text { Scheme S7 and Figure S27 })^{b}\end{array}$ & turquoise & $\begin{array}{l}{[2 \cdot m \mathrm{CPBA}], \mathrm{TS} 2,3, \mathrm{TS} 4,} \\
{[\mathrm{~S} 1 \cdot m \mathrm{CB}], \mathrm{S} 1}\end{array}$ \\
\hline $\begin{array}{l}\text { non-acid-catalyzed exocyclic Criegee } \\
\text { rearrangement (Scheme S8 and } \\
\text { Figure S28) }\end{array}$ & orange $^{c}$ & $\begin{array}{l}{[2 \cdot m \mathrm{CPBA}], \mathrm{TS} 2,3, \mathrm{TS} 5} \\
{[4 \cdot m \mathrm{CB}], 4}\end{array}$ \\
\hline $\begin{array}{l}\text { 1,2,4-trioxane cycloreversion } \\
\text { (Scheme S9 and Figure S29) }\end{array}$ & $\begin{array}{l}\text { dark } \\
\text { green }^{c}\end{array}$ & $\begin{array}{c}{[2 \cdot m \mathrm{CPBA}], \mathrm{TS} 2,3, \mathrm{TS} 6,6,} \\
\text { TS7, }[5 \cdot \text { acetone } \cdot m \mathrm{CBA}]\end{array}$ \\
\hline $\begin{array}{l}\text { 1,2-dioxetane formation (Scheme } \\
\text { S10 and Figure S30) }\end{array}$ & pink & $\begin{array}{l}{[2 \cdot m \mathrm{CPBA}], \text { TS2, } 3, \mathrm{TS} 6,6,} \\
\text { TS8, 7, TS9, [8·mCBA], } \\
\text { 8, TS10 }\end{array}$ \\
\hline $\begin{array}{l}\text { peroxide metathesis (Scheme S11 } \\
\text { and Figure S31) }\end{array}$ & purple $^{c}$ & $\begin{array}{c}{[2 \cdot m \mathrm{CPBA}], \mathrm{TS} 2,3, \mathrm{TS} 11} \\
{[8 \cdot m \mathrm{CBA}], 8, \mathrm{TS} 10}\end{array}$ \\
\hline $\begin{array}{l}\text { tropylium formation (Scheme S12 } \\
\text { and Figure S32) }\end{array}$ & $\operatorname{red}^{c}$ & $\begin{array}{l}9,3,[3 \cdot m \mathrm{CBA}], \mathrm{TS} 3,[4 \cdot \\
2 m \mathrm{CB}], 4\end{array}$ \\
\hline $\begin{array}{l}\text { oxidative epoxide opening (Scheme } \\
\text { S13 and Figure S33) }\end{array}$ & $\begin{array}{l}\text { light } \\
\text { green }^{c}\end{array}$ & $\begin{array}{l}{[2 \cdot m \mathrm{CPBA}], \mathrm{TS} 12,[4 \cdot} \\
m \mathrm{CB}], 4\end{array}$ \\
\hline $\begin{array}{l}\text { concerted reaction (Scheme S14 and } \\
\text { Figure S34) }\end{array}$ & brown $^{c}$ & $\begin{array}{l}{[2 \cdot m \mathrm{CPBA}], \mathrm{TS} 13,[5 \cdot} \\
\text { acetone } \cdot m \mathrm{CBA}]\end{array}$ \\
\hline \multicolumn{3}{|c|}{$\begin{array}{l}{ }^{a} \text { All reaction pathways start with } \mathbf{1},[\mathbf{1} \cdot \mathbf{m C P B A}], \mathbf{T S} \mathbf{1},[\mathbf{2} \cdot \mathbf{m} \mathbf{C B A}] \text {, } \\
\text { and } \mathbf{2} \text { and merge to }[\mathbf{5} \cdot \text { acetone }] \text { and } \mathbf{5} \text {. TS } 1-13=\text { transition states } 1- \\
13, m \mathrm{CPBA}=m e t a-\text { chloroperoxybenzoic acid, } m \mathrm{CB}=\text { meta- } \\
\text { chlorobenzoate, and } m \mathrm{CBA}=m e t a-\text { chlorobenzoic acid. }{ }^{b} \text { This reaction } \\
\text { pathway does not merge to }[\mathbf{5} \cdot \text { acetone }] \text { and } \mathbf{5} .{ }^{c} \text { Reaction pathway is } \\
\text { presented in detail in the Supporting Information, Section } 3 \text {. }\end{array}$} \\
\hline
\end{tabular}

1 was monitored with an offline MS detection using micropillar array ESI, has previously been reported. ${ }^{7}$ Although this study was not a mechanistic investigation, the ion corresponding to $\mathrm{m} / \mathrm{z} 351$ was observed and suggested to be a byproduct. ${ }^{7}$ The online MS experiments of our present study show that the EIP of $m / z 351$ appears to stabilize over the course of the experiments (Figures 1a, S7, S10, and S13), indicating this ion to be a reaction intermediate rather than a byproduct. Based on this, together with the supporting DFT calculations, we now propose structure 4 for the ion $\mathrm{m} / z 351$.

\section{CONCLUSIONS}

We have investigated the mechanism of the $m \mathrm{CPBA}$-mediated oxidation of heptafulvene 1 to tropone 5 (Scheme 1) by combining online MS experiments with a miniaturized reactor and DFT calculations. The mass spectrometrically observed ions provided starting points for the theoretically investigated mechanisms. Based on the energy profiles obtained for each reaction pathway, the most likely pathway (in black and blue) starts with heptafulvene $\mathbf{1}$ initially reacting with a single $m$ CPBA molecule, forming epoxide 2 (Scheme 2). The epoxide oxygen of 2 subsequently reacts with a second equivalent of $m C P B A$. This brings about opening of the epoxide ring (TS2), and by reaction with $m$ CBA, a hemiketal ion (4) is formed. As this ion becomes deprotonated, it forms the tropone product 5 and the byproduct acetone. Thus, the overall reaction could be described as following a Hock-like rearrangement mechanism, ${ }^{27}$ which is known for hydroperoxides with substituents having delocalized electrons.

This study highlights the strength of combining microreactors interfaced with MS with computational chemistry for mechanistic studies and clearly shows that the acid-catalyzed exocyclic Criegee rearrangement pathway is the lowest-energy pathway for $m \mathrm{CPBA}$-mediated oxidation of heptafulvenes to tropones.

\section{EXPERIMENTAL SECTION}

The experimental procedures for coupling the miniaturized reactor to MS for online reaction monitoring and details about the DFT calculations are described here. Details about the MS/MS experiments are presented in the Supporting Information, Section 2.

Reagents for the Online Reaction Monitoring Experiments. The heptafulvene, 8-isopropylidene-4-methoxy-1-methyl-3-trifluoromethyl-8H-benzo $[c d]$ azulene (compound $\mathbf{1}$, Scheme 1 , synthesized as previously described $\left.{ }^{6}\right)$, and meta-chloroperoxybenzoic acid ( $m$ CPBA, $\leq 77 \%$, Sigma-Aldrich, Steinheim, Germany) were used as starting materials for the studied reaction. The compound 5 (4-methoxy-1methyl-3-trifluoromethylbenzo[ $c d]$ azulen-8-one) synthesized as previously described ${ }^{6}$ was used as a reference compound. The following solvents were used for the experiments: Chromasolv-grade acetonitrile (Honeywell, Morris Plains, NJ), formic acid (98-100\%, Merck, Hohenbrunn, Germany), and water (purified with a Milli-Q Plus system from Millipore, Molsheim, France).

Verifying the Purity of the Heptafulvene Reactant 1 and Tropone Product 5 (Reference Compound). We noticed that, in addition to the protonated molecule of 8-isopropylidene-4-methoxy1-methyl-3-trifluoromethyl-8H-benzo $[c d]$ azulene, $[\mathbf{1}+\mathrm{H}]^{+}$, seen at $\mathrm{m} / z 319$, oxidized species of $\mathbf{1}$ were present in electrospray ionization mass spectrometry (ESI-MS) analysis of compound $\mathbf{1}$ (observed as ions at $m / z 293,335$, and 351), using the ion trap mass spectrometer. Due to this, the purity of 1 was investigated by NMR spectroscopy and LC-MS (see further details in the sections NMR Spectroscopy and LC-MS below). The results of these analyses indicate that the oxidation products of $\mathbf{1}$ seen in the direct infusion ESI-MS spectrum are likely due to the oxidation of $\mathbf{1}$ in the ESI process. Reactivity of ESI is a known phenomenon. ${ }^{25}$ When analyzing pure 4-methoxy-1methyl-3-trifluoromethylbenzo[ $c d]$ azulen-8-one (5, synthesized as previously described ${ }^{6}$ ) with ion trap ESI-MS, the only intensive mass peaks present in the mass spectrum were $\mathrm{m} / \mathrm{z} 293$ and 315, which are interpreted as $[5+\mathrm{H}]^{+}$and $[5+\mathrm{Na}]^{+}$, respectively.

NMR Spectroscopy. A $20 \mathrm{mM}$ solution of $\mathbf{1}$ in dimethyl sulfoxide- $d_{6}$ (DMSO- $d_{6}, 100 \%, 99.96$ atom \% D, $0.75 \mathrm{~mL}$; SigmaAldrich, Steinheim, Germany) was placed in a borosilicate glass NMR

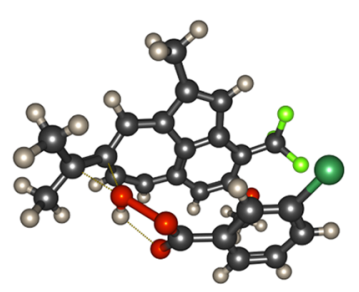

Transition state 1

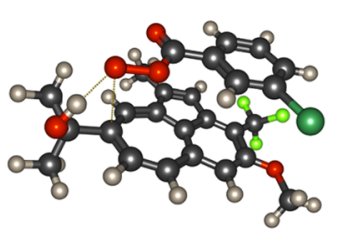

Transition state 2

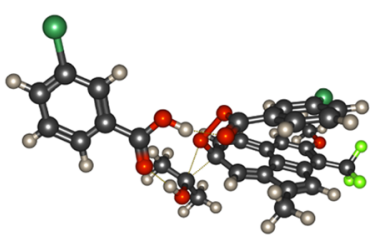

Transition state 3

Figure 3. Optimized structures of the transitions states (TS, also in Figures S60-S62) of the lowest energy barrier pathway, i.e., the blue acidcatalyzed exocyclic Criegee rearrangement pathway, TS1-3, calculated at the M06-2X/6-311++G(d,p) level. 
tube and analyzed with a Bruker Ascend 400-Avance III HD NMR spectrometer (Bruker Corporation, Billerica, MA), operated at the frequency of $400 \mathrm{MHz}$. The chemical shifts $(\delta)$ are given in parts per million (ppm), and the coupling constants are reported in hertz $(\mathrm{Hz})$. The scale of the obtained NMR spectrum was tuned according to the shift of DMSO- $d_{6}(2.5 \mathrm{ppm})$. The NMR data were processed using software MestreNova, version 11.0.3-18688 (Mestrelab Research S.L., Bajo, Spain). The obtained ${ }^{1} \mathrm{H}$ NMR spectrum for compound $\mathbf{1}$ in DMSO- $d_{6}$ is shown in Figure $S 1$.

${ }^{1} \mathrm{H}$ NMR $\left(400 \mathrm{MHz}, \mathrm{DMSO}-d_{6}\right) \delta 6.92(\mathrm{~d}, J=2.0 \mathrm{~Hz}, 1 \mathrm{H}), 6.65$ $(\mathrm{s}, 1 \mathrm{H}), 6.56(\mathrm{~s}, 1 \mathrm{H}), 6.47(\mathrm{dd}, J=12.8,2.0 \mathrm{~Hz}, 1 \mathrm{H}), 6.06(\mathrm{~d}, J=12.8$ $\mathrm{Hz}, 1 \mathrm{H}), 3.83(\mathrm{~s}, 3 \mathrm{H}), 2.16(\mathrm{~d}, J=1.3 \mathrm{~Hz}, 3 \mathrm{H}), 2.00(\mathrm{~s}, 3 \mathrm{H}), 1.94(\mathrm{~s}$, $3 \mathrm{H})$.

LC-MS. A Waters Acquity UPLC system connected to a Waters Synapt G2 mass spectrometer (Waters Corporation, Milford, MA) was used for the measurements. The chromatographic separation was performed with an ACQUITY UPLC BEH C18 column (ID $2.1 \mathrm{~mm}$, length $50 \mathrm{~mm}, 1.7 \mu \mathrm{m}$ particle size). The following eluents were used: A, $100 \%$ water purified with a Milli-Q Plus purification system from Millipore (Molsheim, France); and B, 100\% Chromasolv-grade acetonitrile (Honeywell, Morris Plains, NJ). The solvent gradient started at $95 \%$ solvent A and 5\% solvent B. At 9 min, the portion of B was $95 \%$, which decreased to $5 \%$ at $9.01 \mathrm{~min}$ and remained at this level for the rest of the runtime (total runtime was $10 \mathrm{~min}$ ). The eluent flow rate was $0.6 \mathrm{~mL} / \mathrm{min}$, and the column temperature was kept at $40{ }^{\circ} \mathrm{C}$. One microliter samples of a $31 \mu \mathrm{M}$ solution of 1 and a $34 \mu \mathrm{M}$ solution of $\mathbf{5}$ in Chromasolv-grade acetonitrile were injected to the UPLC. Compound 5 was used as reference compound in the LC-MS experiments to compare its retention time with that of compound 1. The mass spectrometer was operated in positive ESI mode using the following settings: the capillary voltage was $3 \mathrm{kV}$, the ion source temperature $120^{\circ} \mathrm{C}$, and the desolvation temperature 360 ${ }^{\circ} \mathrm{C}$. The measured mass range was $100-600 \mathrm{~m} / z$ for sample 1 and $100-800 \mathrm{~m} / z$ for sample 5 . The scan time of $100 \mathrm{~ms}$ was employed for both samples. Nitrogen was used as the cone gas, as well as desolvation gas. The cone gas flow rate was $10 \mathrm{~L} / \mathrm{h}$ when analyzing 1 and $20 \mathrm{~L} / \mathrm{h}$ when analyzing 5 . The desolvation gas flow rate was 800 $\mathrm{L} / \mathrm{h}$ for both of the samples. The software MassLynx 4.0 (Waters Corporation, Milford, MA) was used to process the acquired data.

The calculated exact mass for the protonated 8-isopropylidene-4methoxy-1-methyl-3-trifluoromethyl-8H-benzo $[c d]$ azulene, $[1+\mathrm{H}]^{+}$, is 319.130 (calculated using MolE Molecular Mass Calculator $\left.\mathrm{v} 2.02^{28}\right)$, and the measured mass for the $[1+\mathrm{H}]^{+}$peak was 319.13 . The calculated exact mass for the protonated 4-methoxy-1-methyl-3trifluoromethylbenzo $[c d]$ azulen-8-one, $[\mathbf{5}+\mathrm{H}]^{+}$, is 293.078 (calculated using MolE Molecular Mass Calculator v2.02 ${ }^{28}$ ), and the measured mass for the $[\mathbf{5}+\mathrm{H}]^{+}$peak was 293.08 . The retention times for $\mathbf{1}$ and $\mathbf{5}$ (in the control sample of $\mathbf{5}$ ) were 7.52 and $5.21 \mathrm{~min}$, respectively (Figure S2). Averaged mass spectra at times when $\mathbf{1}$ and 5 eluted (7.52 and $5.21 \mathrm{~min}$, respectively) are presented in Figure S3. In Figure S3a (analysis of $\mathbf{1}$ ), the main ions observed are at $\mathrm{m} / z$ 293, 319, 335, and 351, while in Figure S3b (analysis of 5), the main ion observed is at $m / z$ 293. In the sample of pure 1 (Figure S3a), the mass peak at $\mathrm{m} / z 319$ corresponds to protonated 8-isopropylidene-4methoxy-1-methyl-3-trifluoromethyl-8H-benzo $[c d]$ azulene, $[1+\mathrm{H}]^{+}$, while the other main mass peaks observed $(\mathrm{m} / z 293,335$, and 351) are interpreted to be oxidation products of 1 . This statement is based on the fact that only one chromatographic peak (at $7.52 \mathrm{~min}$ ) is observed in the chromatogram (Figure S2b) when pure $\mathbf{1}$ is infused. In the mass spectrum in Figure S3b, the only intensive mass peak observed is at $m / z 293$. This is interpreted as $[\mathbf{5}+\mathbf{H}]^{+}$. These results agree with the ones obtained in ESI-MS analysis of these compounds with the ion trap mass spectrometer.

Reagent Solutions for the Oxidation Reaction Experiments. The reactant solutions and the background solvent (a 0.1 vol \% solution of formic acid in acetonitrile) were purged with $\mathrm{N}_{2}$ for $5 \mathrm{~min}$ when they were prepared, as well as when they were sampled (i.e., being drawn into the syringes). Heptafulvene 1 was used as a $31 \mu \mathrm{M}$ $(10.0 \mu \mathrm{g} / \mathrm{mL})$ solution in acetonitrile +0.1 vol \% formic acid. $m$ CPBA was used as a $290 \mu \mathrm{M}(50.0 \mu \mathrm{g} / \mathrm{mL})$ solution in acetonitrile +0.1 vol \% formic acid.

Miniaturized Reactor Fabrication. The miniaturized reactor and the measurement jig for the reactor were $3 \mathrm{D}$ printed with a miniFactory 3D printer (Minifactory Oy Ltd., Seinäjoki, Finland) as described previously. ${ }^{24}$ Briefly, the microreactor was $3 \mathrm{D}$ printed using fused deposition modeling, and the filament used was polypropylene (PP), which exhibits good chemical resistance. The melted PP filament (heated to $230{ }^{\circ} \mathrm{C}$ ) was extruded from a $0.4 \mathrm{~mm}$ diameter nozzle, while the $5 \mathrm{~mm}$ thick PP build platform whereon it was printed was kept at $30{ }^{\circ} \mathrm{C}$. The nano-electrospray ionization (ESI) needle (length $50 \mathrm{~mm}$, i.d. $30 \mu \mathrm{m}$, o.d. $150 \mu \mathrm{m}$; Thermo Fisher Scientific, Vantaa, Finland) and poly(tetrafluoroethylene)-coated stir bar (length $10 \mathrm{~mm}$ ) of the reactor were integrated by pausing the printing process after 28 and 54 layers had been printed, respectively.

Miniaturized Reactor Setup. The miniaturized reactor was coupled to an ion trap mass spectrometer similarly as previously described. ${ }^{24}$ Thus, the measurement jig with the miniaturized reactor was mounted on an XYZ-stage (Märzhäuser Wetzlar GmbH \& Co. $\mathrm{KG}$, Wetzlar, Germany) in front of the capillary extension of an ion trap mass spectrometer (Figure S4). Similarly to in our previous study, a metallic spacer, with a polymer sheet wrapped around it, was placed between the top and bottom part of the measurement jig for the miniaturized reactor to rest on. The power for the mixer fan (in the measurement jig) for activating the stir bar of the reactor was provided by an Iso-Tech DC power supply (IPS 603, Yleiselektroniikka Oyj, Espoo, Finland). The nano-ESI capillary was grounded by wrapping one end of a copper wire around the nano-ESI capillary and the other end of the wire around a metal rod of the ion source frame of the mass spectrometer. Two $1 \mathrm{~mL}$ syringes (i.d. $4.6 \mathrm{~mm}$, Hamilton Company Bonaduz, Bonaduz, Switzerland), placed in a PHD 2000 syringe pump (Harvard Apparatus, Holliston, MA), were used for infusing the reactant solutions. The syringes were connected to the miniaturized reactor in the measurement jig by PEEK capillaries (i.d. $64 \mu \mathrm{m}$, length $42 \mathrm{~cm}$ per capillary; Applied Research Europe $\mathrm{GmbH}$, Berlin, Germany), ferrules, nuts, and sleeves (IDEX Europe GmbH, Erlangen, Germany).

Procedures for Cleaning the Miniaturized Reactor. Between each experiment, the syringes, the PEEK capillaries, and the reactor itself were flushed with at least $1 \mathrm{~mL}$ of acetonitrile. The stir bar of the reactor was activated during the flushing. The capillary extension of the mass spectrometer was sonicated in acetonitrile between each experiment.

Mass Spectrometric Conditions. The mass spectrometer used in the online experiments was an Agilent 6330 ion trap mass spectrometer (Agilent Technologies, Santa Clara, CA) operated in positive ion mode. The ionization technique was electrospray ionization. The $\mathrm{m} / \mathrm{z}$ scan range was $50-600$ in all experiments (including the $\mathrm{MS}^{\mathrm{n}}$ experiments). The capillary voltage was set to $-2.5 \mathrm{kV}$. Nitrogen heated to $100{ }^{\circ} \mathrm{C}$ was used as the drying gas with a flow rate of $6 \mathrm{~L} / \mathrm{min}$. Regarding the online reaction experiments, mass spectra were recorded for at least $34 \mathrm{~min}$ and presented mass spectra of these experiments are averaged over $1 \mathrm{~min}$. Extracted ion profiles (EIPs) of the integer value of the ion of interest were plotted with an isolation width of $\pm 0.5 \mathrm{~m} / z(1.0 \mathrm{~m} / z$ window $)$. The isolation width of all $\mathrm{MS}^{\mathrm{n}}$ experiments was $\pm 0.5 \mathrm{~m} / z(1.0 \mathrm{~m} / z$ window $)$, and the integer value of the precursor ion was isolated in these experiments. $M S^{\mathrm{n}}$ spectra were recorded for $30 \mathrm{~s}$ and averaged over this time. More details about the $M S^{\mathrm{n}}$ experiments (fragmentation amplitudes, relative intensities of reported mass peaks, product ion interpretations) are reported in Table S1 (in Section 2.3.2 of the Supporting Information). The mass spectrometric data were processed with the software DataAnalysis (DataAnalysis for 6300 series ion trap LC/MS version 3.4, build 192; Agilent Technologies, Santa Clara, CA).

Experimental Design of the Online Reaction Monitoring. The online reaction monitoring experiments were conducted at room temperature. Vials, capillaries, syringes, and the reactor were flushed with $\mathrm{N}_{2}$ before each experiment. When starting the experiment, solutions of $m \mathrm{CPBA}$ and $\mathbf{1}$ in acetonitrile $+0.1 \mathrm{vol} \%$ formic acid were contained in one syringe each. The acquisition of mass spectra was 
started at $t=0$, and was continued during the whole experiment. Immediately after the recording of mass spectra was started, $125 \mu \mathrm{L}$ of the contents of each syringe was infused with the flow rate $125 \mu \mathrm{L} /$ min (target volume $125 \mu \mathrm{L}$ ), resulting in filling of the miniaturized reactor (which has the inner volume of approximately $250 \mu \mathrm{L}^{24}$ ) at 1 $\mathrm{min}$. At this time point, the stir bar was activated by turning on the fan in the jig with a voltage of $24 \mathrm{~V}$, and optimization of the position of the reactor in front of the capillary extension of the mass spectrometer was started.

Computational Details. All DFT calculations were carried out with Gaussian 09. ${ }^{29}$ We followed the recommendation of Truhlar et al. $^{30}$ and chose the hybrid functional M06-2X $\mathrm{X}^{31}$ for our calculations. It has been proven to give good results for investigating organic chemistry compounds and reactions. ${ }^{32}$ When employing the functional set with validation sets, the obtained results were much improved compared to the well-established workhorse functional set B3LYP, ${ }^{33-38}$ giving a mean unsigned error (which is the mean absolute deviations from the reference data) of $0.30 \mathrm{kcal} / \mathrm{mol}$ or less. ${ }^{39}$ Owing to its utility for studying hydrocarbon systems, we utilized the basis set $6-311++G(d, p)$ in our calculations. ${ }^{39}$ Aumüller and Yli-Kauhaluoma have studied heptafulvenes resembling the one in our study and concluded that the functional M05-2X (a precursor of M06-2X, which was used in our study) and the basis set 6-311+ $+\mathrm{G}(\mathrm{d}, \mathrm{p})$ yielded accurate results for these systems. ${ }^{40,41}$ All our calculations were conducted in the singlet ground state.

At first, each structure was optimized to its lowest-energy conformation, using the keywords "nosymm" and "UltraFine" for the integration grid. The UltraFine integration grid has 99 radial shells and 590 angular points per shell, making it suitable for calculations on molecules with many tetrahedral centers and larger molecules with many soft modes (such as methyl rotations), which both are characteristics of the systems we studied.

As the mass spectrometry experiments were performed in a $0.1 \mathrm{vol}$ $\%$ solution of formic acid in acetonitrile, all calculations used an integral equation formalism variant polarizable continuum model (IEFPCM) solvent model with the predefined parameters for acetonitrile. Vibrational frequencies of all optimized structures were calculated, yielding no imaginary frequency for stationary structures, and exactly one imaginary frequency for transition states. For (de)protonation steps, no explicit transition states were calculated, and protons were implicitly assumed to originate from meta-chlorobenzoic acid.

\section{ASSOCIATED CONTENT}

\section{S Supporting Information}

The Supporting Information is available free of charge on the ACS Publications website at DOI: 10.1021/acs.joc.9b02078.

NMR spectroscopy results; LC-MS results; miniaturized reactor setup; extracted ion profiles; mass spectra; fragmentation table and schemes; DFT calculations; optimized structures; Gibbs free energies, enthalpies, and entropies; electronic energies, zero-point energies, and entropies; Cartesian coordinates (PDF)

\section{AUTHOR INFORMATION}

\section{Corresponding Author}

*E-mail: jari.yli-kauhaluoma@helsinki.fi.

\section{ORCID $\odot$}

Sofia M. E. Nilsson: 0000-0003-2696-1742

Henning Henschel: 0000-0001-7196-661X

Tapio Kotiaho: 0000-0003-0382-8578

Jari Yli-Kauhaluoma: 0000-0003-0370-7653

\section{Notes}

The authors declare no competing financial interest.

\section{ACKNOWLEDGMENTS}

Computational resources were granted by the CSC-IT Center for Science in Espoo, Finland. Nina Sipari is acknowledged for the LC-MS measurements. Financial support was provided by the Academy of Finland (projects 276627, 257316, and 307464) and the Doctoral Program in Drug Research, University of Helsinki.

\section{REFERENCES}

(1) Nakagawa, Y.; Tayama, K. Mechanism of Mitochondrial Dysfunction and Cytotoxicity Induced by Tropolones in Isolated Rat Hepatocytes. Chem.-Biol. Interact. 1998, 116, 45-60.

(2) Kiriazis, A.; Vahakoski, R. L.; Santio, N. M.; Arnaudova, R.; Eerola, S. K.; Rainio, E.-M.; Aumüller, I. B.; Yli-Kauhaluoma, J.; Koskinen, P. J. Tricyclic Benzo $[c d]$ azulenes Selectively Inhibit Activities of Pim Kinases and Restrict Growth of Epstein-Barr Virus-Transformed Cells. PLoS One 2013, 8, No. e55409.

(3) Trust, T. J.; Bartlett, K. H. Antibacterial Activity of Tropilidine and Tropone. Antimicrob. Agents Chemother. 1975, 8, 381-383.

(4) Barbachyn, M. R.; Toops, D. S.; Ulanowicz, D. A.; Grega, K. C.; Brickner, S. J.; Ford, C. W.; Zurenko, G. E.; Hamel, J. C.; Schaadt, R. D.; Stapert, D.; et al. Synthesis and Antibacterial Activity of New Tropone-Substituted Phenyloxazolidinone Antibacterial Agents 1. Identification of Leads and Importance of the Tropone Substitution Pattern. Bioorg. Med. Chem. Lett. 1996, 6, 1003-1008.

(5) Donlin, M. J.; Zunica, A.; Lipnicky, A.; Garimallaprabhakaran, A. K.; Berkowitz, A. J.; Grigoryan, A.; Meyers, M. J.; Tavis, J. E.; Murelli, R. P. Troponoids Can Inhibit Growth of the Human Fungal Pathogen Cryptococcus neoformans. Antimicrob. Agents Chemother. 2017, 61, No. e02574.

(6) Aumüller, I. B.; Yli-Kauhaluoma, J. Benzo $[c d]$ azulene Skeleton: Azulene, Heptafulvene, and Tropone Derivatives. Org. Lett. 2009, 11, $5363-5365$.

(7) Nissilä, T.; Backman, N.; Kolmonen, M.; Leinonen, A.; Kiriazis, A.; Yli-Kauhaluoma, J.; Sainiemi, L.; Kostiainen, R.; Franssila, S.; Ketola, R. A. Rotating Multitip Micropillar Array Electrospray Ionization-Mass Spectrometry for Rapid Analysis and HighThroughput Screening. Int. J. Mass Spectrom. 2012, 310, 65-71.

(8) Liu, Y.; Jiang, X. Why Microfluidics? Merits and Trends in Chemical Synthesis. Lab Chip 2017, 17, 3960-3978.

(9) Jensen, K. F.; Reizman, B. J.; Newman, S. G. Tools for Chemical Synthesis in Microsystems. Lab Chip 2014, 14, 3206-3212.

(10) Fabris, D. Mass Spectrometric Approaches for the Investigation of Dynamic Processes in Condensed Phase. Mass Spectrom. Rev. 2005, $24,30-54$.

(11) Glish, G. L.; Vachet, R. W. The Basics of Mass Spectrometry in the Twenty-First Century. Nat. Rev. Drug Discovery 2003, 2, 140-150.

(12) Yan, X.; Sokol, E.; Li, X.; Li, G.; Xu, S.; Cooks, R. G. On-Line Reaction Monitoring and Mechanistic Studies by Mass Spectrometry: Negishi Cross-Coupling, Hydrogenolysis, and Reductive Amination. Angew. Chem., Int. Ed. 2014, 53, 5931-5935.

(13) Bain, R. M.; Sathyamoorthi, S.; Zare, R. N. "On-Droplet" Chemistry: The Cycloaddition of Diethyl Azodicarboxylate and Quadricyclane. Angew. Chem., Int. Ed. 2017, 56, 15083-15087.

(14) Bain, R. M.; Pulliam, C. J.; Ayrton, S. T.; Bain, K.; Cooks, R. G. Accelerated Hydrazone Formation in Charged Microdroplets. Rapid Commun. Mass Spectrom. 2016, 30, 1875-1878.

(15) Cai, Y.; Wang, J.; Zhang, Y.; Li, Z.; Hu, D.; Zheng, N.; Chen, H. Detection of Fleeting Amine Radical Cations and Elucidation of Chain Processes in Visible-Light-Mediated $[3+2]$ Annulation by Online Mass Spectrometric Techniques. J. Am. Chem. Soc. 2017, 139, 12259-12266.

(16) Ray, A.; Bristow, T.; Whitmore, C.; Mosely, J. On-Line Reaction Monitoring by Mass Spectrometry, Modern Approaches for the Analysis of Chemical Reactions. Mass Spectrom. Rev. 2018, 37, $565-579$.

(17) De Souza, R. O. M. A.; da Penha, E. T.; Milagre, H. M. S.; Garden, S. J.; Esteves, P. M.; Eberlin, M. N.; Antunes, O. A. The 
Three-Component Biginelli Reaction: A Combined Experimental and Theoretical Mechanistic Investigation. Chem. - Eur. J. 2009, 15, 9799-9804.

(18) Alvim, H. G. O.; Bataglion, G. A.; Ramos, L. M.; de Oliviera, A. L.; de Oliviera, H. C. B.; Eberlin, M. N.; de Macedo, J. L.; da Silva, W.

A.; Neto, B. A. D. Task-Specific Ionic Liquid Incorporating Anionic Heteropolyacid-Catalyzed Hantzsch and Mannich Multicomponent Reactions. Ionic Liquid Effect Probed by ESI-MS(/MS). Tetrahedron 2014, 70, 3306-3313.

(19) Rodrigues, T. S.; Silva, V. H. C.; Lalli, P. M.; de Oliveira, H. C. B.; da Silva, W. A.; Coelho, F.; Eberlin, M. N.; Neto, B. A. D. MoritaBaylis-Hillman Reaction: ESI-MS(/MS) Investigation with Charge Tags and Ionic Liquid Effect Origin Revealed by DFT Calculations. J. Org. Chem. 2014, 79, 5239-5248.

(20) Jašíková, L.; Anania, M.; Hybelbauerová, S.; Roithová, J. Reaction Intermediates Kinetics in Solution Investigated by Electrospray Ionization Mass Spectrometry: Diaurated Complexes. J. Am. Chem. Soc. 2015, 137, 13647-13657.

(21) Zhou, S.; Li, J.; Schlangen, M.; Schwarz, H. Bond Activation by Metal-Carbene Complexes in the Gas Phase. Acc. Chem. Res. 2016, 49, 494-502.

(22) Godoi, M. N.; de Azambuja, F.; Martinez, P. D. G.; Morgon, N. H.; Santos, V. G.; Regiani, T.; Lesage, D.; Dossmann, H.; Cole, R. B.; Eberlin, M. N.; et al. Revisiting the Intermolecular Fujiwara Hydroarylation of Alkynes. Eur. J. Org. Chem. 2017, 2017, 17941803.

(23) Schwarz, H. Ménage-à-Trois: Single-Atom Catalysis, Mass Spectrometry, and Computational Chemistry. Catal. Sci. Technol. 2017, 7, 4302-4314.

(24) Scotti, G.; Nilsson, S. M. E.; Haapala, M.; Pöhö, P.; Boije af Gennäs, G.; Yli-Kauhaluoma, J.; Kotiaho, T. A Miniaturised 3D Printed Polypropylene Reactor for Online Reaction Analysis by Mass Spectrometry. React. Chem. Eng. 2017, 2, 299-303.

(25) Van Berkel, G. J.; Kertesz, V. Using the Electrochemistry of the Electrospray Ion Source. Anal. Chem. 2007, 79, 5510-5520.

(26) Farahani, P.; Roca-Sanjuán, D.; Zapata, F.; Lindh, R. Revisiting the Nonadiabatic Process in 1,2-Dioxetane. J. Chem. Theory Comput. 2013, 9, 5404-5411.

(27) Yaremenko, I. A.; Vil', V. A.; Demchuk, D. V.; Terent'ev, A. O. Rearrangements of Organic Peroxides and Related Processes. Beilstein J. Org. Chem. 2016, 12, 1647-1748.

(28) MolE Molecular Calculator v. 2.02; https://mods.rna.albany. edu/masspec/MoIE (accessed February 20, 2018).

(29) Frisch, M. J.; Trucks, G. W.; Schlegel, H. B.; Scuseria, G. E.; Robb, A.; Cheeseman, J. R.; Scalmani, G.; Barone, V.; Menuccci, B.; Peterson, G. A.; et al. Gaussian 09; Gaussian, Inc.: Wallingford, CT, 2009.

(30) Zhao, Y.; Truhlar, D. G. Density Functionals with Broad Applicability in Chemistry. Acc. Chem. Res. 2008, 41, 157-167.

(31) Zhao, Y.; Truhlar, D. G. The M06 Suite of Density Functionals for Main Group Thermochemistry, Thermochemical Kinetics, Noncovalent Interactions, Excited States, and Transition Elements: Two New Functionals and Systematic Testing of Four M06-Class Functionals and 12 Other Functionals. Theor. Chem. Acc. 2008, 120, 215-241.

(32) Zhao, Y.; Truhlar, D. G. Applications and Validations of the Minnesota Density Functionals. Chem. Phys. Lett. 2011, 502, 1-13.

(33) Zhao, Y.; Truhlar, D. G. A Density Functional That Accounts for Medium-Range Correlation Energies in Organic Chemistry. Org. Lett. 2006, 8, 5753-5755.

(34) Wodrich, M. D.; Corminboeuf, C.; Schreiner, P. R.; Fokin, A. A.; Schleyer, P. v. R. How Accurate Are DFT Treatments of Organic Energies? Org. Lett. 2007, 9, 1851-1854.

(35) Rokob, T. A.; Hamza, A.; Pápai, I. Computing Reliable Energetics for Conjugate Addition Reactions. Org. Lett. 2007, 9, $4279-4282$.

(36) Zhao, Y.; Truhlar, D. G. How Well Can New-Generation Density Functionals Describe Protonated Epoxides Where Older Functionals Fail? J. Org. Chem. 2007, 72, 295-298.
(37) Zhao, Y.; Truhlar, D. G. How Well Can New-Generation Density Functionals Describe the Energetics of Bond-Dissociation Reactions Producing Radicals? J. Phys. Chem. A 2008, 112, 10951099.

(38) Steinmann, S. N.; Wodrich, M. D.; Corminboeuf, C. Overcoming Systematic DFT Errors for Hydrocarbon Reaction Energies. Theor. Chem. Acc. 2010, 127, 429-442.

(39) Peverati, R.; Truhlar, D. G. Quest for a Universal Density Functional: The Accuracy of Density Functionals across a Broad Spectrum of Databases in Chemistry and Physics. Philos. Trans. $R$. Soc., A 2014, 372, No. 20120476.

(40) Aumüller, I. B.; Yli-Kauhaluoma, J. Computational Methods for Analysis of an Unsaturated Carbocycle: Heptafulvene. Theor. Chem. Acc. 2010, 126, 55-73.

(41) Aumüller, I. B.; Yli-Kauhaluoma, J. Angle Strain and Conjugation in Conformations of Heptafulvenes. Comput. Theor. Chem. 2011, 964, 40-48. 